13,18

\title{
Электронная и магнитная структура интеркалированных пленок графена
}

\author{
(C) С.М. Дунаевский ${ }^{1,2}$, Е.Ю. Лобанова ${ }^{3,4}$, Е.К. Михайленко ${ }^{3,4}$, И.И. Пронин ${ }^{3}$ \\ ${ }^{1}$ Национальный исследовательский центр „Курчатовский институт“, \\ Петербургский институт ядерной фризики им. Б.П. Константинова, \\ Гатчина, Россия \\ ${ }^{2}$ Санкт-Петербургский государственный электротехнический университет „ЛЭТИ“ им. В.И. Ульянова (Ленина), \\ Санкт-Петербург, Россия \\ ${ }^{3}$ Физико-технический институт им. А.Ф. Иофффе РАН, \\ Санкт-Петербург, Россия \\ ${ }^{4}$ Санкт-Петербургский политехнический университет Петра Великого, \\ Санкт-Петербург, Россия \\ E-mail: smd2000@mail.ru
}

В рамках теории спинового функционала плотности (SDFT) выполнены ab initio расчеты электронного спектра системы графен-кобальт-никель. В работе представлены дисперсионные кривые $E_{\sigma n}(\mathbf{k})$, на основе которых определены парциальные и полные плотности состояний валентных электронов, вычислены значения магнитных моментов всех атомов в суперячейке. Показано, что энергетическое положение „конуса Дирака“, обусловленного $p_{z}$-состояниями графена, слабо зависит от числа слоев Со, интеркалированного в межслоевой зазор между кобальтом и графеном.

DOI: $10.21883 /$ FTT.2018.06.46000.08M

\section{1. Введение}

Уникальные свойства графена (Gr) позволяют рассматривать его в качестве одного из наиболее перспективных материалов современной спинтроники [1-4]. Для практического использования графена необходимо понимать, как модифицируется его электронная структура при взаимодействии с различными подложками. В частности, применение графена неизбежно ведет к необходимости создания контакта графен/металл или графен/изолятор. Модификация свойств таких гетероструктур выполняется путем интеркаляции системы графен/подложка атомами других веществ. Так, интеркаляция графена переходными металлами перспективна для изготовления туннельных магнитных переходов и структур графен/ферромагнитный металл/подложка с перпендикулярной магнитной анизотропией. Ввиду того, что формирование ферромагнитных слоев под графеном представляет особый интерес для развития спинтроники, интеркаляция пленок графена (на никеле) железом, кобальтом и кремнием в последние годы стала объектом активных экспериментальных и теоретических исследований $[5,6]$.

Одним из наиболее популярных интерфейсов графен/металл является система $\mathrm{Gr} / \mathrm{Ni}(111)$ [7]. Это обусловлено тем, что в силу малого отличия постоянных решетки графена и никеля высококачественный графен может быть получен на поверхности $\mathrm{Ni}(111)$ методом химического осаждения из газовой фазы (CVD). Теоретическое и экспериментальное исследование графена на никеле показало, что при контакте с никелем электронная структура графена значительно модифицируется. Это обусловлено гибридизацией $d$-состояний никеля с $s$ - и $p$-состояниями углерода, которая разрушает линейную дисперсию состояний вблизи точки $K$. Интеркаляция системы $\mathrm{Gr} / \mathrm{Ni}(111)$ атомами железа исследовалась в работах $[8,9]$. Ранее, однако, „из первых принципов“ теоретически был рассмотрен только случай интеркаляции системы $\mathrm{Gr} / \mathrm{Ni}(111)$ одним монослоем (ML) железа. В работе [10], где мы изучили эволюцию электронного строения интерфейса $\mathrm{Gr} / \mathrm{Ni}(111)$ в процессе его интеркаляции железом, этот диапазон был расширен до пяти монослоев. Так как нам неизвестны расчеты электронной структуры $\mathrm{Gr} / \mathrm{Ni}(111)$, интеркалированной кобальтом, то в настоящей работе впервые представ-

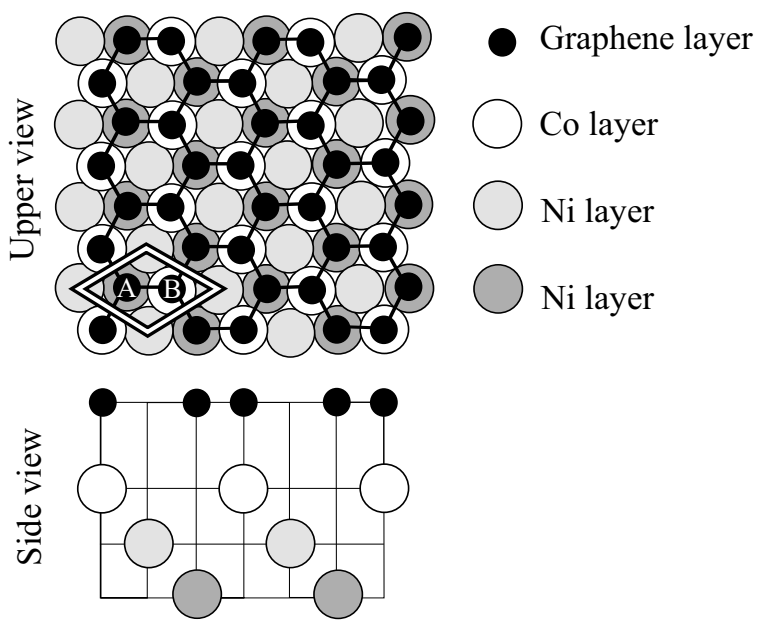

Рис. 1. Атомная структура систем $\mathrm{Gr} / \mathrm{Co} / \mathrm{Ni}(111)$ и $\mathrm{Gr} / \mathrm{Fe} / \mathrm{Ni}(111)$. На видах сверху выделены „поверхностные“ элементарные ячейки. Показаны атомы углерода подрешеток $A$ и $B$. 

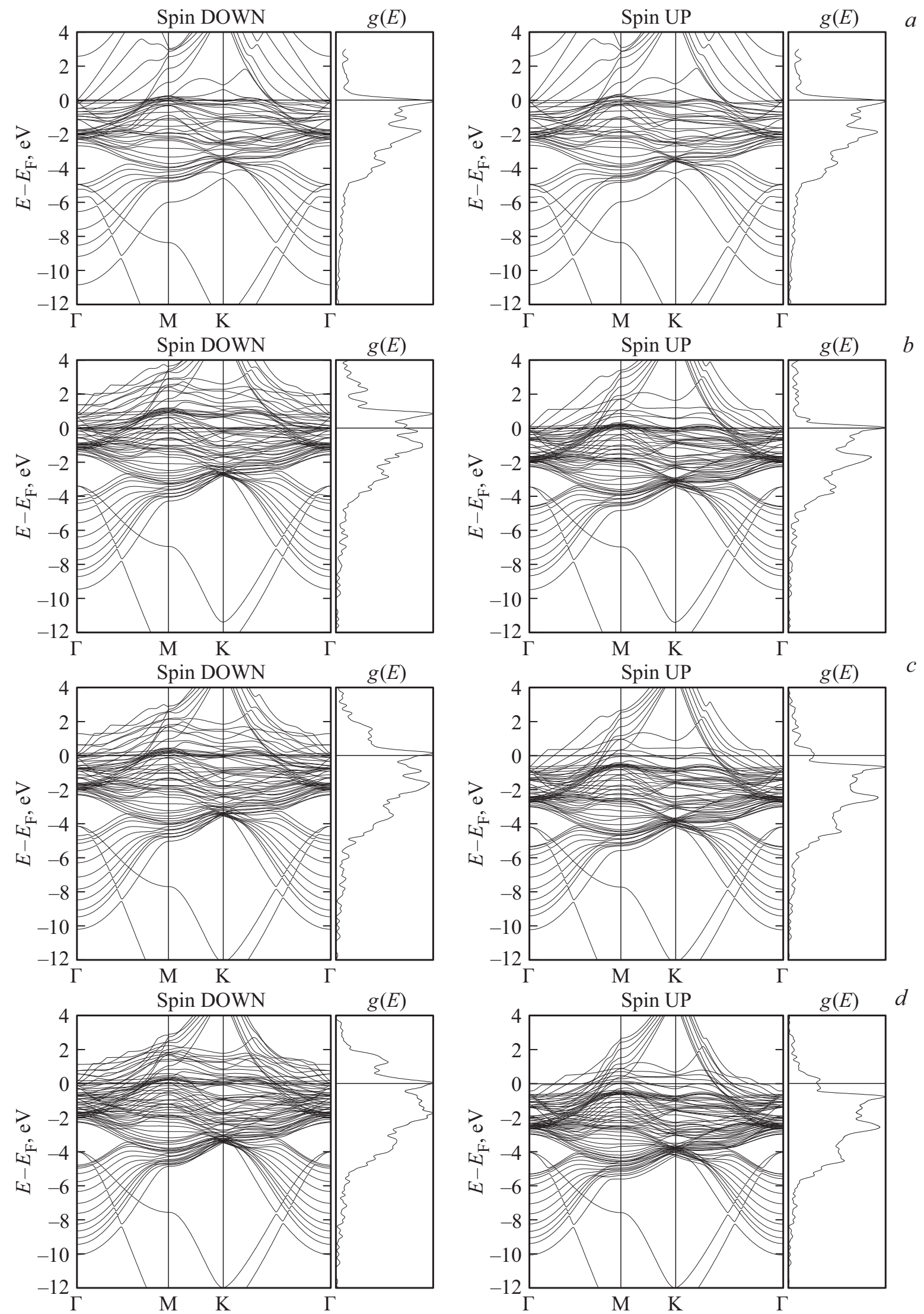

Рис. 2. Спектр $E_{\sigma n}(\mathbf{k})$ и плотность состояний (DOS) систем: (a) - $\mathrm{Gr} / \mathrm{Ni}(111), \quad(b)-\mathrm{Gr} / 1 \mathrm{MLCo} / \mathrm{Ni}(111)$, (c) $\mathrm{Gr} / 3 \mathrm{MLCo} / \mathrm{Ni}(111),(d)-\mathrm{Gr} / 5 \mathrm{MLCo} / \mathrm{Ni}(111)$. 


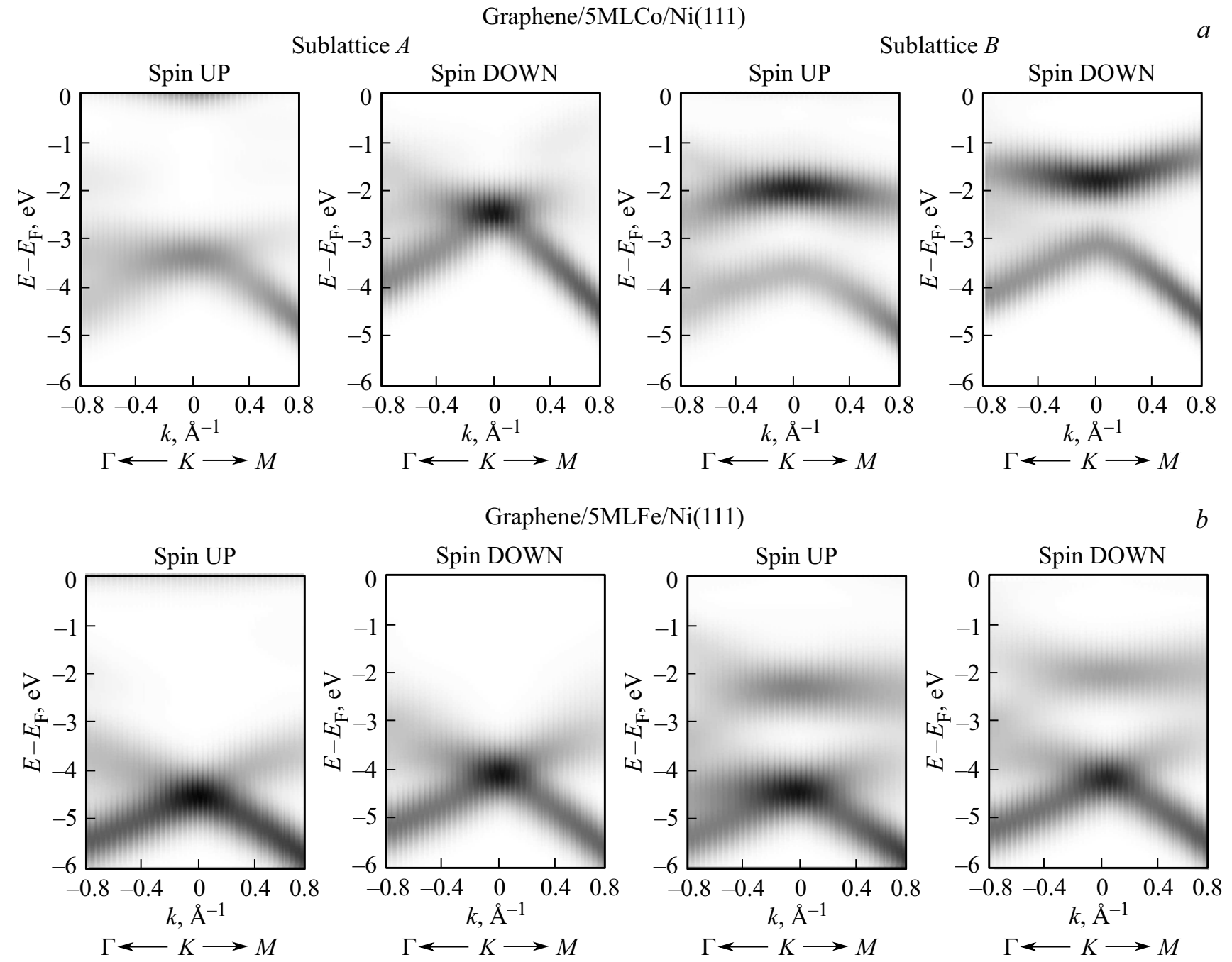

Рис. 3. Вклады $p_{z}$-состояний в спектр $E_{\sigma n}(\mathbf{k})$ двух подрешеток углерода для: $(a)-\mathrm{Gr} / 5 \mathrm{MLCo} / \mathrm{Ni}(111),(b)-\mathrm{Gr} / 5 \mathrm{MLFe} / \mathrm{Ni}(111)$.

лены спектры $E_{\sigma n}(\mathbf{k})$, полные (DOS) и парциальные (PDOS) плотности состояний, позволяющие находить числа заполнения атомных орбиталей в системе, атомные магнитные моменты и полные энергии системы $\mathrm{Gr} / \mathrm{Co} / \mathrm{Ni}(111)$.

\section{2. Метод расчета}

$A b$ initio расчеты были выполнены в рамках метода псевдопотенциала, реализованного в пакете Quantum Espresso [11] (PWscf). В расчетах использовалось обобщенное градиентное разложение (GGA) и псевдопотенциалы PBE (см. [11]). Максимальная энергия плоских волн в разложения волновых функций была выбрана равной $200 \mathrm{Ry}$. В ходе самосогласованных вычислений применялось Monkhorst-Pack разбиение зоны Бриллюэна на $7 \times 7 \times 2$ точек. Начальные конфигурации валентных электронов имели вид: $2 s^{2} p^{2}(\mathrm{C}), 3 s^{2} p^{6} 3 d^{8} 4 s^{2}(\mathrm{Ni})$, $3 s^{2} p^{6} 3 d^{7} 4 s^{2}$ (Со). Для моделирования поверхности $\mathrm{Ni}(111)$ использовалась двумерная гексагональная решетка Браве с постоянной, равной $2.42 \AA$, показанная на рис. 1. На виде сверху выделены „поверхностные элементарные ячейки, а также показаны атомы углерода подрешеток $A$ и $B$. В работе [10] нами было показано, что именно для такого расположения пленки графена на никеле полная энергия системы минимальна.

Суперячейка включала в себя семь атомов никеля, от одного до пяти атомов кобальта (по одному атому на слой) и два атома углерода. Расстояние между верхним слоем атомов кобальта и графеном было принято равным 2.07 А.. Ширина вакуумного зазора равнялась $14 \AA$. Для всех рассмотренных систем была выполнена структурная оптимизация.

\section{3. Результаты и их обсуждение}

На рис. 2 представлены результаты расчета спектра $E_{\sigma n}(\mathbf{k})$ и полной плотности состояний систем $\mathrm{Gr} / \mathrm{Co} / \mathrm{Ni}(111)$ для двух проекций спина $g_{\sigma}(E)$. Peзультаты расчета зонной структуры исходной системы $\mathrm{Gr} / \mathrm{Ni}(111)$ показаны на рис. 2, $a$. Результаты, иллю- 

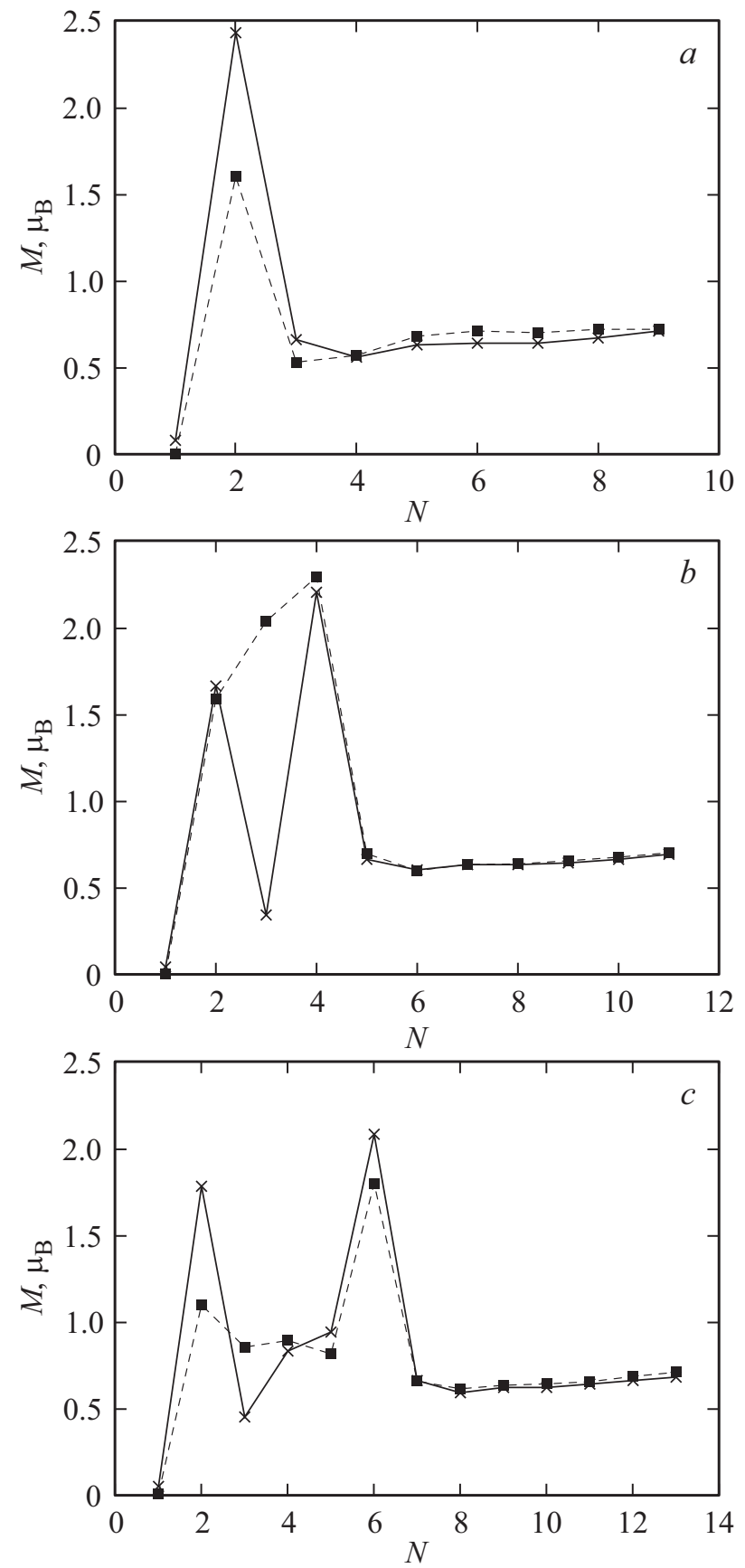

Рис. 4. Атомные магнитные моменты для атомов разных слоев систем $\mathrm{Cr} / \mathrm{Co} / \mathrm{Ni}(111)$ (крестики) и $\mathrm{Cr} / \mathrm{Fe} / \mathrm{Ni}(111)$ (квадраты): (a) $-1 \mathrm{MLCo}(\mathrm{Fe}),(b)-3 \mathrm{MLCo}(\mathrm{Fe}),(c)-5 \mathrm{MLCo}(\mathrm{Fe})$. Магнитные моменты углерода не превышают значения $0.05 \mu_{B}$. Число атомов $\mathrm{Ni}$, моделирующих подложку, во всех случаях равнялось семи.

стрирующие эволюцию энергетической структуры системы с интеркаляцией атомов кобальта, приведены на рис. $2, b-d$. За начало отсчета по шкале энергий принята энергия Ферми $E_{\mathrm{F}}$. Из сопоставления зонной структуры систем $\mathrm{Gr} / \mathrm{Co} / \mathrm{Ni}(111)$ и $\mathrm{Gr} / \mathrm{Ni}(111)$ видно, что разница между дисперсионными кривыми, построенными для разных проекций спина, незаметная для системы
$\mathrm{Gr} / \mathrm{Ni}(111)$, становится существенной при внедрении атомов кобальта и особенно заметно проявляется вблизи уровня Ферми. В результате перекрытия р-орбиталей атомов углерода с $3 d$-орбиталями металла вблизи уровня Ферми существует большое число заполненных гибридизованных зон, что и обеспечивает основные изменения энергетической структуры при увеличении числа интеркалированных слоев кобальта. При выполнении численных расчетов предполагалось, что при интеркаляции атомов кобальта в межслоевое пространство между никелем и графеном получающиеся пленки кобальта повторяют структуру подложки $\mathrm{Ni}(111)$.

Для каждого значения $E_{\sigma n}(\mathbf{k})$ может быть рассчитан вклад в соответствующую полную волновую функцию $\psi_{\sigma n}(\mathbf{k})$ той или иной атомной орбитали. В первую очередь всех интересует вопрос существования „конуса Дирака“, обусловленного вкладом $p_{z}$-орбиталей углерода. В качестве примера на рис. 3 показаны соответствующие вклады $p_{z}$-орбиталей поверхностных атомов углерода из двух подрешеток графена в зонную структуру вблизи точки $K$ систем $\mathrm{Gr} / 5 \mathrm{MLCo} / \mathrm{Ni}(111)$ и $\mathrm{Gr} / 5 \mathrm{MLFe} / \mathrm{Ni}(111)$. Атомы углерода подрешетки $A$ находятся в положении „fсc“ относительно атомов подложки, атомы подрешетки $B-$ в положении „tор“. Видно, что гибридизация состояний углерода и металлов проявляется сильнее для атомов углерода, находящихся непосредственно над атомами подложки (подрешетка $B$ ). При этом разница между плотностями для двух проекций спина проявляется сильнее для системы $\mathrm{Gr} / \mathrm{Co} / \mathrm{Ni}(111)$. На рисунке можно видеть подобие „конусов Дирака“ вблизи точки $K$, которые из-за взаимодействия для разных проекций спина оказываются сдвинуты относительно друг друга. Качественно, вклад от $p_{z}$-орбиталей согласуется с экспериментальными картинами фотоэлектронной спектроскопии с угловым разрешением (ФЭСУР). Для системы $\mathrm{Gr} / \mathrm{Fe} / \mathrm{Ni}$ данные ФЭСУР представлены в [12]. Из спектров $E_{\sigma n}(\mathbf{k})$ и DOS нами были вычислены числа заполнения всех атомных орбиталей в системе и найдены атомные магнитные моменты $\mathrm{Co}$, Ni и углерода. На рис. 4 показаны атомные магнитные моменты для всех слоев системы $\mathrm{Gr} / \mathrm{Ni}(111)$, интеркалированной одним $(a)$, тремя $(b)$ и пятью $(c)$ монослоями Со (крестики) или $\mathrm{Fe}$ (квадратики). Магнитные моменты углерода для всех систем не превышают значения $0.05 \mu_{B}$. Магнитные моменты на семи атомах никеля колеблются относительно известного значения для объема, равного $0.63 \mu_{B}$. Для всех систем наблюдается увеличение магнитного момента интеркалированных атомов, находящихся в контакте с атомами никеля.

\section{4. Заключение}

В настоящей работе впервые рассмотрено изменение электронного спектра и атомных магнитных моментов системы $\mathrm{Gr} / \mathrm{Co} / \mathrm{Ni}(111)$ с ростом числа $N$ монослоев кобальта, интеркалированных в межслоевое пространство графен-никель. Для $N=1-5 \mathrm{ML}$ Cо представлены 
$a b$ initio спин-поляризованные дисперсионные кривые $E_{\sigma n}(\mathbf{k})$, соответствующие плотности состояний валентных электронов, локальные магнитные моменты никеля, кобальта и графена в случае их ферромагнитного упорядочения. Кроме того, нами выделен вклад $p_{z}$-состояний графена в спектр $E_{\sigma n}(\mathbf{k})$. Форма этих вкладов для спиновых подзон вблизи точки $K$ зоны Бриллюэна напоминает искаженный „конус Дирака“, вершина которого находится значительно ниже уровня Ферми. Для состояний со спином „вверх“ конус сдвигается к бо́льшим энергиям связи по сравнению с состояниями со спином „вниз“. Это свидетельствует о том, что интеркаляция графена кобальтом приводит к появлению конечной спиновой поляризации электронных состояний углерода. Обнаружено, что в интеркалированной пленке кобальта (железа) наибольшими магнитными моментами обладают атомы, непосредственно контактирующие с графеном и никелем.

Численные расчеты проведены с использованием вычислительных ресурсов суперкомпьютерного центра СПбПУ.

\section{Список литературы}

[1] A.K. Geim, K.S. Novoselov. Nature Mater. 6, 183 (2007).

[2] J. Wintterlin, M.-L. Bocquet. Surf. Sci. 603, 1841 (2009).

[3] J. Ryu, Y. Kim, D. Won, N. Kim, J.S. Park, E.-K. Lee, D. Cho, S.-P. Cho, S.J. Kim, G.H. Ryu, H.-A.-S. Shin, Z. Lee, B.H. Hong, S. Cho. ACS Nano 8, 950 (2014).

[4] A. Varykhalov, J. Sanchez-Barriga, D. Marchenko, P. Hlawenka, P.S. Mandal, O. Rader, Nature Commun. 6, 7610 (2015).

[5] M. Weser, E.N. Voloshina, K. Horn, Y.S. Dedkov. Phys. Chem. Chem. Phys. 13, 7534 (2011).

[6] N. Rougemaille, A.T. N'Diaye, J. Coraux, C. Vo-Van, O. Fruchart, A.K. Schmid. Appl. Phys. Lett. 101, 142403 (2012).

[7] G. Bertoni, L. Calmels, A. Altibelli, V. Serin. Phys. Rev. B 71, 075402 (2004)

[8] Yu.S. Dedkov, A.M. Shikin, V.K. Adamchuk, S.L. Molodtsov, C. Laubschat, A. Bauer, G. Kaindl. Phys. Rev. B 64, 035405 (2001).

[9] C. Riedl, C. Coletti, T. Iwasaki, A.A. Zakharov, U. Starke. Phys. Rev. Lett. 103, 246804 (2009).

[10] И.И. Пронин, С.М. Дунаевский, Е.Ю. Лобанова, Е.К. Михайленко. ФТТ 59, 10, 2037 (2017).

[11] P. Giannozzi, S. Baroni, N. Bonini, M. Calandra, R. Car, C. Cavazzoni, D. Ceresoli, G.L. Chiarotti, M. Cococcioni, I. Dabo, A.D. Corso, S. de Gironcoli, S. Fabris, G. Fratesi, R. Gebauer, U. Gerstmann, C. Gougoussis, A. Kokalj, M. Lazzeri, L. Martin-Samos, N. Marzari, F. Mauri, R. Mazzarello, S. Paolini, A. Pasquarello, L. Paulatto, C. Sbraccia, S. Scandolo, G. Sclauzero, A.P. Seitsonen, A. Smogunov, P. Umari, R.M. Wentzcovitch. J. Phys.: Condens. Matter 21, 395502 (2009).

[12] G.S. Grebenyuk, O.Yu. Vilkov, A.G. Rybkin, M.V. Gomoyunova, B.V. Senkovskiy, D.Yu. Usachov, D.V. Vyalikh, S.L. Molodtsov, I.I. Pronin. Appl. Surf. Sci. 392, 715 (2017).

Публикация материалов Конференции завершена. 